\title{
Monitoring the visual field of a patient with secondary pseudotumour cerebri syndrome due to one-sided lymphadenectomy and internal jugular vein ligature - case report
}

\author{
Beata Gajda', Agnieszka Kiszka', Edyta Koman', Agnieszka Cisek², \\ Katarzyna Nowomiejska ${ }^{1}$, Robert Rejdak ${ }^{1}$ \\ 'Department of General Ophthalmology, Medical University of Lublin, Poland \\ ${ }^{2}$ Department of Human Anatomy, Medical Faculty University of Rzeszow, Poland
}

\begin{abstract}
We describe a case of a 37-year-old man who developed prolonged papilledema as a result of increased intracranial pressure following unilateral radical neck dissection due to diffuse large B-cell non-Hodgkin lymphoma. It seems that the increase in intracranial pressure was a result of insufficient collateral venous drainage of the brain. Clinical examination showed bilateral papilloedema, diplopia, and visual deterioration in the left eye. Magnetic resonance imaging and computed tomography of the brain were normal. Semi-automated kinetic perimetry was used to monitor the visual function during 12 months of the follow-up. This method revealed enlargement of the blind spot in the left eye without progression. Peripheral borders of the visual field were within normal limits.
\end{abstract}

KEY WORDS: peripheral visual field, pseudotumour cerebri, lymphadenectomy, jugular vein

Ophthalmol J 2016; Vol. 1, No. 2, 67-71

\section{INTRODUCTION}

Pseudotumour cerebri syndrome (PTCS) refers to the primary and secondary disorders that cause elevated intracranial pressure without a central nervous system infection, intracranial mass lesion, or malignancy. Secondary PTCS results from a recognised medical condition, medication toxicity, or venous abnormality, which leads to elevated intracranial pressure. The most feared complication of PTCS is vision loss due to papilledema, but headache is the most frequent symptom. The main targets of treatment are reduction of symptoms and preservation of vision [1].

Semi-automated kinetic perimetry (SKP) provides a method for assessment of the peripheral visual field with up to 90 degrees of eccentricity us- ing Goldmann stimuli moved with constant angular velocity. The development of SKP examination resulted in greater standardisation, consistency, analysis, reliability, and interpretation of kinetic visual field (VF) results in neuroophthalmology [2].

We describe a case of a 37-year-old man who developed prolonged papilledema as a result of increased intracranial pressure following unilateral radical neck dissection and ligation of the internal jugular vein. Visual function was monitored during the follow-up period using SKP.

\section{CASE REPORT}

A 37-year-old patient was referred to our clinic due to bilateral papilledema lasting for nine months 


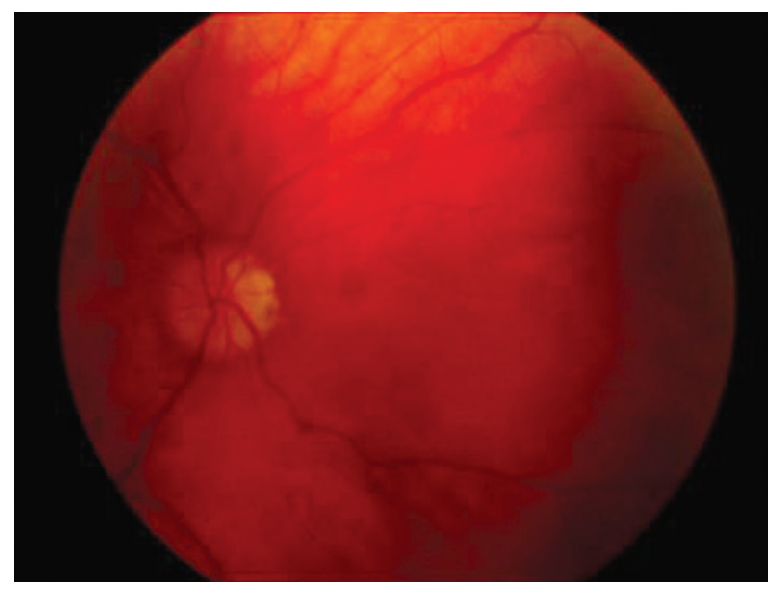

FIGURE 1. Photograph of the fundus of the left eye showing papilloedema

OD

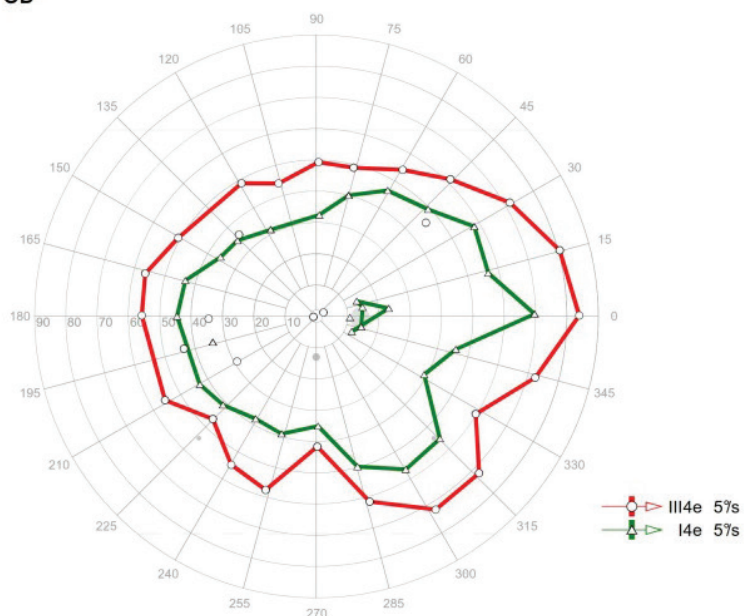

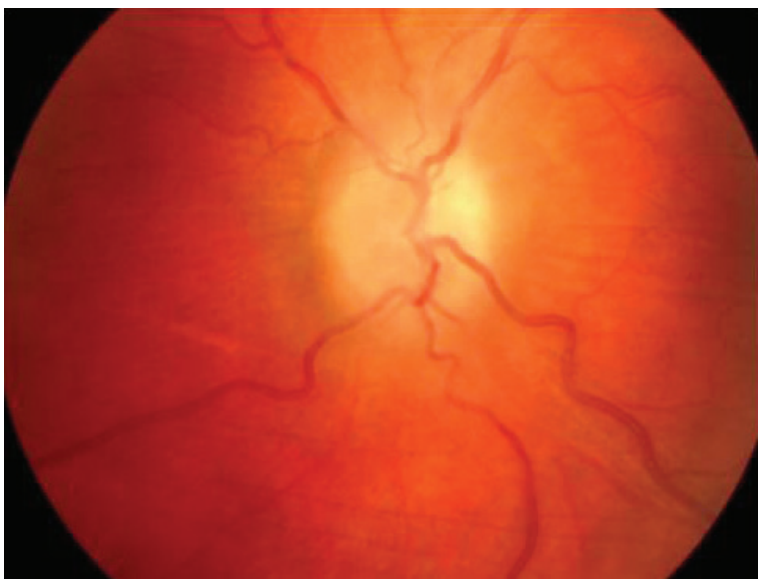

FIGURE 2. Photograph of the fundus of the right eye showing papilloedema

os

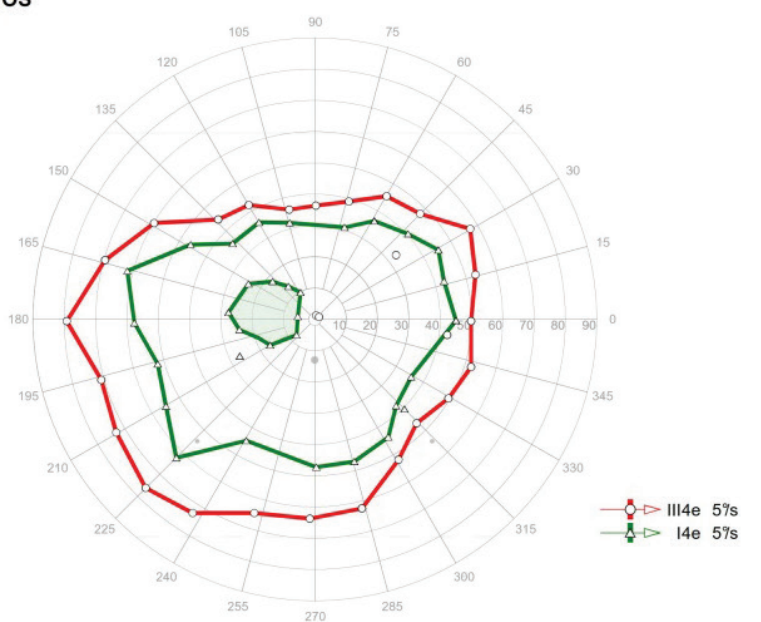

FIGURE 3. Semi-automated kinetic perimetry examination (SKP) of both eyes (right eye on the left and left eye on the right) during the first examination. Two isopters (III4e and I4e) are used to assess the visual field. There is enlargement of the blind spot in the left eye

(Fig. 1-2). The first symptoms appeared two weeks after surgical resection of neck tumour on the right side (unilateral modified radical neck dissection). The patient suffered from diffuse large B-cell non-Hodgkin lymphoma. He presented then with a history of diplopia and visual impairment of the left eye. Best-corrected visual acuity (BCVA) at that time was 1.0 in the right eye and 0.6 in the left eye. Intraocular pressure and anterior segment examination presented no deviations. Laryngological and internal medicine examinations of the patient did not show any abnormalities. Computer tomography (CT) of the brain was normal.

The mobility of the eyeballs and the reaction of the pupils to light and colour vision were correct. Fundus examination showed bilateral papilledema and choroidal folds in the left eye. BCVA of the right eye was 1.0 and of the left eye 0.4 .

SKP (Octopus 900, Haag Streit, Switzerland) of both eyes was performed two times during 12 months of the follow-up. Two different stimuli were used: III4e and I4e. Blind spot was assessed using I4e stimulus. In both examinations it showed enlargement of the blind spot in the left eye (Fig. 3-4). Peripheral borders of VF were within normal limits. Optical coherence tomography (OCT) showed thickened peripapillary nerve fibre layers (RNFL).

Neurological consultation showed increased intracranial pressure. The magnetic resonance imaging (MRI) of the brain was normal. During the follow-up period of 12 months we observed a stable condition of the patient without disease progression. 

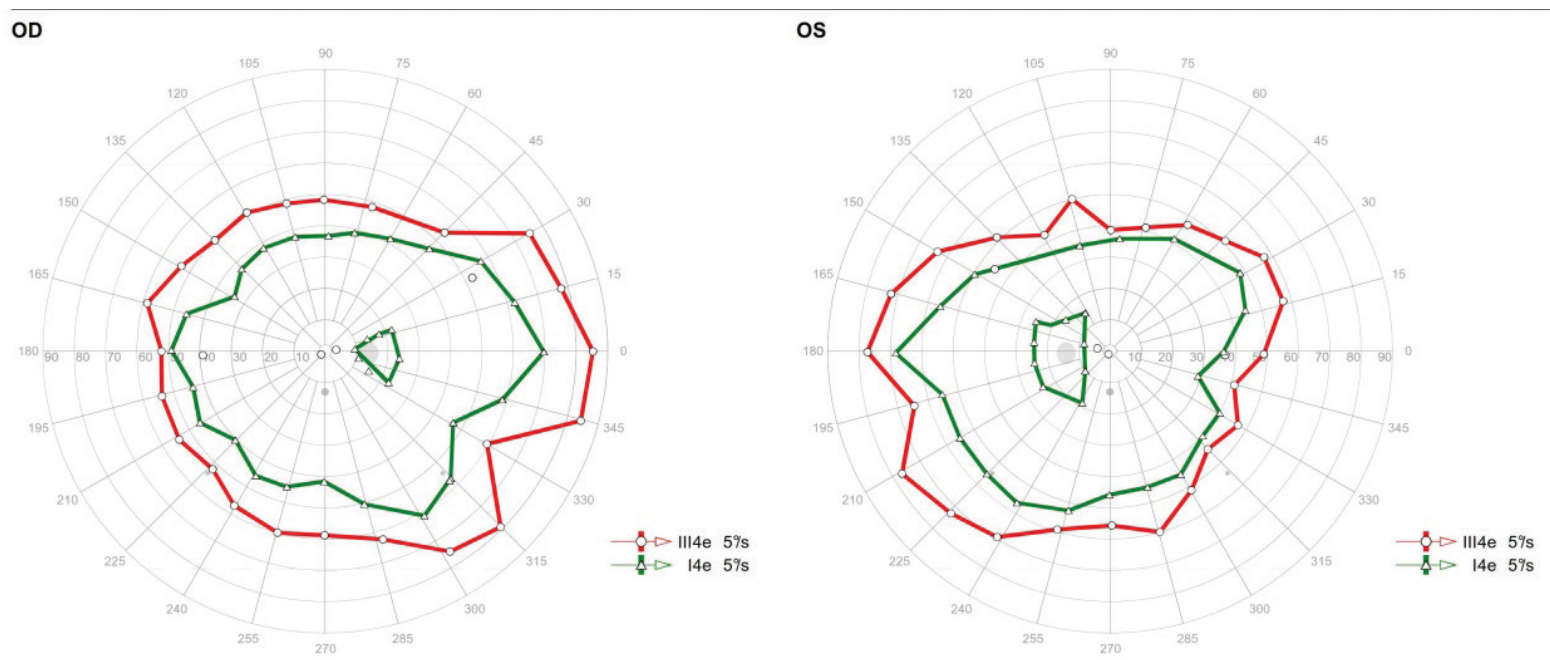

FIGURE 4. Semi-automated kinetic perimetry examination (SKP) of both eyes (right eye on the left and left eye on the right) during the second examination. Two isopters (III4e and $14 \mathrm{e}$ ) are used to assess the visual field. There is enlargement of the blind spot in the left eye

\section{DISCUSSION}

We report a case of a patient who developed symptoms of secondary PTCS after unilateral lymphadenectomy operation and ligation of the internal jugular vein. It seems that an increase in intracranial pressure was a result of insufficient collateral venous drainage of the brain.

From inside the cranium venous blood can flow out through the veins and venous plexuses located in the holes and canals of the base of the skull, ocular veins, diploic veins, and emissary veins $[3,4]$. The most important venous vessels providing blood outflow from the cavity of the skull are the internal jugular veins, which are often irregular in diameter (with a majority share of the left internal jugular vein). The outflow of blood through the internal and external vertebral venous plexuses and deep cervical veins is also very important $[4,5]$.

Principal outflow through the internal jugular veins can be substituted through communications at the cranial base by the large vertebral plexuses. Depending on the availability of venous collateral circulation of the brain, the closure of one or both of the internal jugular veins may block the outflow of venous blood from the brain. Simultaneous obstruction of principal and collateral venous outflow will result in an increase in venous pressure and eventual insufficiency of cerebral blood flow (CBF). This will cause increased intracranial pressure [4].

In cases of unilateral radical lymphadenectomy with removal of the jugular vein, the opposite internal jugular vein takes blood drainage from the brain. If there are anomalies in the venous sinuses, in the other internal jugular vein [6], or in other components of collateral circulation [7], symptoms of increased intracranial pressure may occur in patients who have undergone unilateral radical lymphadenectomy.

Recently updated criteria for the diagnosis of PTCS require: papilledema, normal neurologic examination aside from intracranial nerve abnormalities, neuroimaging findings including no abnormal meningeal enhancement on MRI examination and regular brain parenchyma, normal CSF composition, and elevated opening pressure in the lumbar puncture ( $\geq 250 \mathrm{~mm}$ CSF in adults and $\geq 280 \mathrm{~mm}$ CSF in children). If the patient does not present with papilloedema, the diagnosis can be suggested if three of the following neuroimaging findings are presented additionally: flattening of the posterior aspect of the globe, empty sella, distention of the perioptic subarachnoid space with or without a tortuous optic nerve or transverse venous sinus stenosis [8].

The most common manifestation, which occurs in $75-99 \%$ of patients with PTCS, is headache. It is non-specific and is usually holocranial [9]. Other typical signs include papilloedema, visual field defects, and sixth nerve palsy. Common symptoms are diplopia, visual loss, and pulsatile tinnitus [10].

This disease should be differentiated from Idiopathic Intracranial Hypertension (IIH), i.e. the primary form of PTCS. It occurs mainly in obese women of childbearing age. The most important diseases that predispose to secondary PTCS include: polycystic ovarian syndrome (PCOS), Addison disease, Pickwickian syndrome, Down and Turner dis- 
ease, Sydenham's chorea, anaemia, renal failure, or venous thrombosis. Drugs and other substances (vitamin A, antibiotics, hormones, and lithium), blood and connective tissue disorders, increased protein level in the CSF, and rapid growth in infancy can also contribute to disease expansion $[1,11]$.

To this day, thirteen cases of increased intracranial pressure after unilateral radical lymphadenectomy have been published in the literature [7, 12-17]. Eleven of them concerned left-sided lymphadenectomy. The time between surgery and the development of symptoms was between three days and two months. Visual disturbances and headache were the most common symptoms. Diplopia occurred in six patients. Other symptoms such as nausea, vomiting, and tinnitus occurred less frequently.

Our patient developed diplopia and impairment of vision. Those symptoms appeared 14 days after right-sided lymphadenectomy, without deviations in CT and MRI examinations.

There is no explicit test that would be recommended for monitoring the treatment of patients with PTCS. Among imaging tests that can be performed are CT, MRI, and MRA (magnetic resonance angiography). Those examinations are usually performed in patients with suspected PTCS [18]. SD-OCT is a noninvasive imaging test that allows us to evaluate the thickness of the retina around the optic nerve and macula. OCT is necessary when we observe even a slight swelling of the optic nerve head. It can detect small changes in the optic nerve when no other changes are observed. This test is used both to recognise changes in the optic nerve and to monitor the effectiveness of treatment of a disease $[19,20]$.

In our patient we used SKP for assessment of the entire VF. This method revealed enlargement of the blind spot in the left eye without progression. Manual kinetic perimetry is the method of choice in cases of advanced VF deficits in neuro-ophthalmic diseases. Kinetic testing is more sensitive for detecting peripheral VF defects, less time-consuming, and more efficient for detection and monitoring progression of large VF defects compared with conventional automated static perimetry. Additionally, it provides more interaction between the examiner and the patient during testing [2]. However, there is the need for a skilled technician, difficulties in quantifying area of isopters, challenges in defining serial changes over time, and a lack of standardisation of stimulus direction and speed that can introduce human bias.
SKP has restored interest among physicians in kinetic perimetry. This examination provides the advantages of automated serial VF examinations, including the ability to present following tests in an identical way [21].

SKP allows computer-controlled standardised presentation over the entire VF, for any chosen Goldmann stimulus size, intensity combination, and in any direction. In addition, the starting and ending points for the stimulus vectors are predefined, and there is a constant angular velocity [2].

All patient's responses and stimulus characteristics can be processed and are stored in an electronic database. SKP examination enhances stimulus presentation, selected by the examiner, with a constant angular velocity and standardisation and reproducibility by computer-controlled auto-calibration [22].

Another advantage of kinetic testing using SKP is the capacity to assess the reaction time (RT) for the subject for each VF session. On the basis of the individual RT of the subject we can correct the position of the response [2].

SKP has been already used in monitoring the VF of patients with neuro-ophthalmic diseases as haemianopia [23], optic nerve head drusen [24], and Vigabatrin-related VF constriction [25].

\section{CONCLUSIONS}

Secondary PTCS due to unilateral radical neck dissection is a rare entity. SKP is a method for monitoring the course of the disease in patients with PTCS. Using this method, we can effectively assess changes of VF in the patient over time.

\section{REFERENCES}

1. Mallery RM, Friedman DI, Liu GT. Headache and the pseudotumor cerebri syndrome. Curr Pain Headache Rep 2014; 18: 446.

2. Grobbel J, Dietzsch J, Johnson CA et al. Normal values for the full visual field, Corrected for Age- and Reaction Time, Using Semiautomated Kinetic Testing on the Octopus 900 Perimeter. TransI Vis Sci Technol 2016; 5: 5 .

3. Iskra T. Fizjologia krążenia mózgowego. In: Szczudlik A, Członkowska A, Kwieciński H, Stowik A (eds.) Udar mózgu. Kraków 2007: 3-25.

4. Andeweg J. The anatomy of collateral venous flow from the brain and its value in aetiological interpretation of intracranial pathology. Neuroradiology 1996; 38: 621-628.

5. Schaller B. Physiology of cerebral venous blood flow: from experimental data in animals to normal function in humans. Brain Res Rev 2004; 46: 243-260.

6. Karaman E, Saritzali G, Cansiz H. A case of increased intracranial pressure after unilateral modified radical neck dissection. Am J Otolaryngol 2009; 30: 261-263.

7. Doepp F, Schreiber SJ, Benndorf G, Radtke A, Gallinat J, Valdueza $\mathrm{JM}$. Venous drainage patterns in a case of pseudotumor cerebri following unilateral radical neck dissection. Acta Otolaryngol 2003; 123: 994-997. 
8. Baykan B, Ekizoğlu E, Altıokka Uzun G. An update on the pathophysiology of idiopathic intracranial hypertension alias pseudotumor cerebri. J Turkish Soc Algol 2015; 27: 63-72.

9. Kosmorsky GS. Idiopathic intracranial hypertension: Pseudotumor cerebri. Headache 2014; 54: 389-393.

10. Thurtell MJ, Wall M. Idiopathic intracranial hypertension: Recognition, treatment, and ongoing management. Curr Treat Options Neurol 2013; 15: 1-12.

11. Balmaceda CM. Guzy. In: Kwieciński H, Kamińska A (eds.). Neurologia Merritta. Urban \& Partner, Wroclaw 2004: 299-390.

12. Fitz-Hugh GS, Robins RB, Craddock WD. Increased intracranial pressure complicating unilateral neck dissection. Laryngoscope 1996; 76: 893-906.

13. H. M. Morfit. Permanent increased intracranial pressure following unilateral radical neck dissection. Arch Surg 1958; 76: 713.

14. Marr WG, Chambers RG. Pseudotumor cerebri syndrome following unilateral radical neck dissection. Am J Ophthalmol 1961; 51: 605-611.

15. Blervacque A, Béal F, Malbrel P. Papilledema after an operation at the level of the neck. Bull des sociétés d'ophtalmologie Fr 1965; 65: 135-136.

16. Tobin HA. Increased cerebrospinal fluid pressure following unilateral radical neck dissection. Laryngoscope 1972; 82: 817-820.

17. Hunt MG, Lee AG, Kardon RH, Lesley WS, Chaloupka JC. Improvement in papilledema and visual loss after endovascular stent placement in dural sinus thrombosis. Neuroophthalmology 2001; 26: 85-92.

18. Degnan AJ, Levy LM. Pseudotumor cerebri: brief review of clinical syndrome and imaging findings. Am J Neuroradiol 2011; 32: 1986-1993.
19. Marzoli SB, Ciasca P, Curone M et al. Quantitative analysis of optic nerve damage in idiopathic intracranial hypertension $(\mathrm{IIH})$ at diagnosis. Neurol Sci 2013; 34 (suppl. 1): S143-A145.

20. Skau M, Yri H, Sander B, Gerds TA, Milea D, Jensen R. Diagnostic value of optical coherence tomography for intracranial pressure in idiopathic intracranial hypertension. Graefe's Arch Clin Exp Ophthalmol 2013; 251: 567-574.

21. Ramirez AM, Chaya CJ, Gordon LK, Giaconi JA. A comparison of semiautomated versus manual Goldmann kinetic perimetry in patients with visually significant glaucoma. J Glaucoma 2008; 17: 111-117.

22. Nevalainen J, Paetzold J, Krapp E, Vonthein R. The use of semi-automated kinetic perimetry (SKP) to monitor advanced glaucomatous visual field loss. Graefes Arch Clin Exp Ophthalmol 2008; 246: 1331-1339.

23. Nowomiejska K, Vonthein R, Paetzold J, Zagorski Z, Kardon R, Schiefer $U$. Comparison between semiautomated kinetic perimetry and conventional Goldmann manual kinetic perimetry in advanced visual field loss. Ophthalmology 2005; 112: 1343-1354.

24. Nowomiejska K, Rejdak R, Zagorski Z, Zarnowski T. Comparison of static automated perimetry and semi-automated kinetic perimetry in patients with bilateral visible optic nerve head drusen. Acta Ophthalmol 2009; 87: 801-805.

25. Nowomiejska K, Jedrych M, Brzozowska A et al. Relationship between the area of isopters and Vigabatrin dosage during two years of observation. BMC Ophthalmol 2014; 14: 56. 
